\title{
Review of: "Medical Factors Associated With Fracture Nonunion: A Retrospective Study"
}

\author{
Ryogo Furuhata
}

Potential competing interests: The author(s) declared that no potential competing interests exist.

In this study, the authors retrospectively identified the risk factors of postoperative nonunion in clavicle fractures and femur fractures using multivariate analyses. This study is new in that it also evaluates hematological examination of patients as potential risk factors for postoperative nonunion of fractures. This manuscript is written in very easy-to-understand English. The statistical analysis used in this study is also considered valid. However, there are several major questions in the research design of this study.

1) I think radiographical assessment is important for bone healing of fractures in addition to patient background and hematologic examination, but radiological assessment is not included as an explanatory variable. For example, differences in fracture type, such as transverse versus oblique fractures, or fracture site, such as distal clavicle versus midshaft fractures, may have a significant effect on fracture bone healing. Without the addition of these variables in univariate and multivariate analyses, it is impossible to assess whether hematological or radiological data have a greater impact on fracture healing.

2) I would like to know why this study forced on the two completely different types of fractures: clavicle fractures and femur fractures. Since the amount of preoperative blood loss associated with fracture is different between femur and clavicle fractures, are the blood test data different between the two fractures? In that case, it would be better to separate the results for each fracture type.

In addition, it would be easier for the readers to understand the interpretation of the results if the following points are clarified

1) The results identifying fibrinogen levels and lymphocyte counts as risk factors for nonunion are very interesting, but what is the cutoff value? Deriving it from the ROC curve would be a more significant clinical message.

2) I would like to know the possible mechanisms by which fibrinogen levels and lymphocyte counts are risk factors for nonunion. Has this been discussed in the past literature? If there is any discussion of such mechanisms in the past literature, it would be helpful to add it to the manuscript. 